\title{
SISTEMAS E CONDIÇÕES DE COLHEITA E ARMAZENAMENTO NA QUALIDADE DE MORANGOS CV. CAMAROSA ${ }^{1}$
}

\author{
MARCELO BARBOSA MALGARIM², RUFINO FERNANDO FLORES CANTILLANO², ENILTON FICK COUTINHO²
}

\begin{abstract}
RESUMO - O objetivo deste trabalho foi avaliar a qualidade de morangos 'Camarosa' submetidos a diferentes sistemas de colheita, luz UV-C, atmosfera modificada e armazenamento por diferentes períodos, visando ao aumento da vida útil e à redução de perdas após a colheita. A colheita foi realizada em dois sistemas: no sistema um, o produtor realizou a colheita sem luvas, em caixas de madeira e sem utilizar o pré-resfriamento das frutas; no sistema dois, a colheita foi realizada por pessoal treinado, com utilização de luvas desinfetadas, em caixas plásticas lavadas e desinfetadas, foi empregado o pré-resfriamento a $-3^{\circ} \mathrm{C}$ durante 1 hora, até a temperatura da polpa das frutas atingir $4^{\circ} \mathrm{C}$. Os tratamentos foram: T1controle (frutas sem luz UV-C e sem filme de polietileno); T2- frutas submetidas à luz UV-C durante 6 minutos; T3- frutas acondicionadas em filme de polietileno; T4- frutas submetidas à luz UV-C e acondicionadas em filme de polietileno. $\mathrm{O}$ armazenamento foi realizado à temperatura de $0 \pm 0,5^{\circ} \mathrm{C}$ e UR de 90-95\% por períodos de 3; 6 e 9 dias, seguidos de simulação da comercialização por 3 dias, à temperatura de $8 \pm 0,5^{\circ} \mathrm{Ce} \mathrm{UR} \mathrm{de} 75-80 \%$. Na colheita e após o armazenamento, avaliaram-se: perda de massa; cor; firmeza de polpa; sólidos solúveis (SS); acidez total titulável (ATT); relação SS/ATT; ácido ascórbico, e incidência de podridões. A utilização de pré-resfriamento reduziu a perda de massa e a porcentagem de podridões. A perda de massa diminuiu nas frutas tratadas com filme. A luz UV-C reduziu a porcentagem de podridões. Morangos 'Camarosa' colhidos cuidadosamente, submetidos a pré-resfriamento, com utilização de luz UV-C e filme de polietileno, mantêm a qualidade durante nove dias de armazenamento a $0^{\circ} \mathrm{C}$ e 3 dias de comercialização a $8^{\circ} \mathrm{C}$.
\end{abstract}

Termos para indexação: Pré-resfriamento, atmosfera modificada, luz UV-C, qualidade.

\section{SYSTEMS AND CONDITIONS OF HARVEST AND STORAGE IN STRAWBERRIES CV'S CAMAROSA QUALITY}

ABSTRACT - The objective of this work was to evaluate the cv. Camarosa's quality of strawberries submitted to different harvest systems, UV$\mathrm{C}$ light, exposure modified atmosphere and storage for different periods, seeking the increase of the useful life and the reduction of losses after the crop. The harvest was accomplished in two systems, in system one the producer accomplished the harvest without gloves, into wood boxes and without using the pre-cooling of the fruits. In system two the harvest was accomplished by trained people, who used disinfected gloves, and put the strawberries into also disinfected and washed plastic boxes, the pre-coolig which was used was $-3 \mathrm{C}$ for 1 hour, up to the fruits pulp temperature reached 4C. The following treatments were tested: T1- control (fruits without UV-C light and without polyethylene bag); T2- fruits submitted to UV-C light for 6 minutes; T3- fruits conditioned polyethylene bag; T4- fruits submitted to the UV-C light and conditioned under a polyethylene bag. The storage was accomplished under a temperature of $0 \pm 0,5 \mathrm{C}$ and 90 to $95 \%$ relative humidity (RH) through periods of 3,6 and 9 days followed by commercialization simulation for 3 days under the temperature of $8 \pm 1 \mathrm{C}$ and RH between 75 to $80 \%$. In the harvest and after the storage it was evaluated weight loss; color; pulp firmness; soluble solids (SS); titratable acidity (TA); SS/TA ratio; ascorbic acid and incidence of rottenness. The pre-cooling usage reduced the weight loss and the percentage of rottenness. The weight loss decreased in treated fruits that was covered with polyethylene The UV-C light reduced the percentage of rottenness. Cv. Camarosa strawberries picked carefully, submitted to the pre-cooling, that went under the UV-C light and polyethylene film, maintains the quality for nine days of storage under $0^{\circ} \mathrm{C}$ and 3 days of commercialization to $8^{\circ} \mathrm{C}$.

Index terms: Pre-cooling, modified atmosphere, UV-C light, strawberry quality.

\section{INTRODUÇÃO}

Com a mudança dos hábitos alimentares ocorridos nos últimos anos no País, e com maior exigência de qualidade por parte dos consumidores, começa a haver também maior necessidade de utilização da refrigeração, não somente para a melhoria da qualidade como também para a redução das perdas.

O morango apresenta elevada perecibilidade pós-colheita, principalmente devido a sua intensa atividade metabólica e grande suscetibilidade ao ataque de agentes patogênicos causadores de podridões. A utilização de baixas temperaturas é essencial para o préresfriamento, armazenamento, transporte a longas distâncias e comercialização de morangos. Entretanto, para o armazenamento prolongado, somente a redução da temperatura não é suficiente para manter a qualidade das frutas, sendo necessário usar também outras técnicas visando a prolongar a vida útil dos frutos.

Atualmente, existe a preocupação dos consumidores com possíveis resíduos agroquímicos nas frutas, estimulando, desta forma, o estudo de métodos alternativos para a redução de pragas e doenças. Agentes químicos atuam sobre os patógenos de ferimentos ou sobre aqueles que apresentam infecção quiescente e possuem a grande vantagem de seu efeito residual garantir proteção durante o armazenamento prolongado dos frutos (Benato et al., 2001). Entretanto, a crescente restrição ao uso de fungicidas, por questões de segurança alimentar e impacto ambiental, tem estimulado o uso de métodos alternativos e biológicos para o controle de doenças (Binotti et al., 2002).

A indução da resistência no controle de fitopatógenos em pós-colheita, por processos naturais, é um estudo crescente e que vem alcançando resultados promissores nos últimos anos (Benato, 2003). Marquenie et al., (2002) verificaram redução do desenvolvimento de fungos em morangos com o uso de luz UV-C. Segundo Neves et al. (2002), o uso de embalagens de polietileno de baixa densidade, associada ao AR, preserva a integridade das frutas, possibilitando melhor manutenção dos atributos sensoriais das mesmas. Segundo Wszelaki \& Mitcham (2003), a combinação de vários tratamentos póscolheita, inclusive com morangos, tem sido estudada com resultados promissores.

O objetivo deste trabalho foi avaliar a qualidade de morangos 'Camarosa' submetidos a diferentes sistemas de colheita, luz UV-C, atmosfera modificada e armazenamento por diferentes períodos, visando ao aumento da vida útil após a colheita e à redução de perdas.

\section{MATERIAL E MÉTODOS}

Morangos 'Camarosa' foram colhidos no estádio maduro,

\footnotetext{
(Trabalho 162-2005). Recebido: 05-10-2005. Aceito para publicação: 30-05-2006.

${ }^{2}$ Eng. Agr., Dr. Professor do Departamento de Fitotecnia da Universidade Estadual de Ponta Grossa - UEPG/PR. malgarim@yahoo.com.

${ }^{3}$ Eng. Agr., Dr. Pesquisador da Embrapa Clima Temperado, Pelotas/RS. fcantil@cpact.embrapa.br / enilton@cpact.embrapa.br.
} 
em dois sistemas de colheita, em um produtor comercial da região de Pelotas-RS. No sistema um (S1), o produtor realizou a colheita de forma usual (sem luvas, em caixas de madeira e sem utilização de préresfriamento), e no sistema dois (S2), a colheita foi realizada por pessoal treinado, com utilização de luvas desinfetadas e caixas plásticas de colheita lavadas e desinfetadas, e, logo após a colheita, as frutas foram submetidas a pré-resfriamento a $-3^{\circ} \mathrm{C}$, durante 1 hora, até a temperatura da polpa atingir $4^{\circ} \mathrm{C}$ (Figura 1$)$.

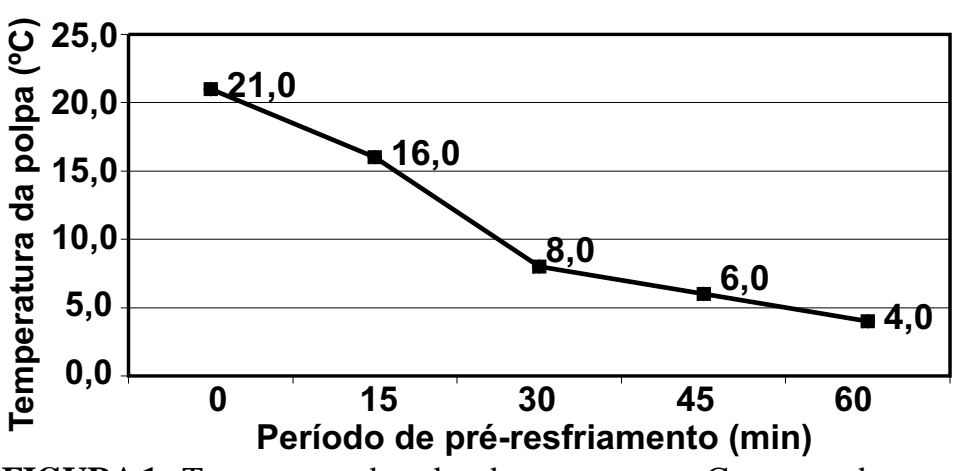

FIGURA 1 - Temperatura da polpa de morangos cv. Camarosa durante o pré-resfriamento em câmara fria à temperatura de $-3^{\circ} \mathrm{C}$.

As frutas colhidas nos dois sistemas foram selecionadas e receberam os tratamentos: T1- controle (frutas sem luz UV-C e sem filme de polietileno); T2- frutas submetidas à luz UV-C durante 6 minutos, com $25 \mathrm{~cm}$ de distância da lâmpada; T3- frutas acondicionadas em filme de polietileno, com $7 \mu$ de espessura por parede; T4- frutas submetidas à luz UV-C e acondicionadas em filme de polietileno.

$\mathrm{O}$ armazenamento refrigerado foi realizado à temperatura de $0 \pm 0,5^{\circ} \mathrm{C}$ e UR de $90-95 \%$ por três, seis e nove dias seguidos de simulação da comercialização por três dias à temperatura de $8 \pm 0,5^{\circ} \mathrm{Ce}$ UR de $75-80 \%$.

$\mathrm{Na}$ colheita e após o armazenamento, as análises das frutas foram realizadas no laboratório de Pós-colheita da Embrapa Clima Temperado, onde se avaliaram as variáveis: a) perda de massa: calculada a partir das diferenças de peso das unidades experimentais observadas entre o momento da instalação do experimento e a avaliação de controle de qualidade após o armazenamento, sendo os resultados expressos em porcentagem (\%); b) cor de superfície e de fundo: medida com duas leituras em lados opostos na região equatorial das frutas. As leituras foram realizadas com colorímetro Minolta CR300, com fonte de luz D 65, com $8 \mathrm{~mm}$ de abertura. No padrão C.I.E. $\mathrm{L}^{*} \mathrm{a}^{*} \mathrm{~b}^{*}$, onde os valores $\mathrm{a}^{*}, \mathrm{~b}^{*}$ são usados para calcular o ângulo Hue ou matiz $\left({ }^{\circ} \mathrm{h}^{*}=\operatorname{tang}^{-1} \mathrm{~b}^{*} \cdot \mathrm{a}^{*-1}\right)$; c) firmeza de polpa (FP): medida com penetrômetro manual McCornick FT 327 com ponteira de 5/16 polegadas de diâmetro, realizando-se duas leituras em lados opostos da secção equatorial das frutas, expressas em Newton (N); d) sólidos solúveis (SS): por refratometria, realizada com um refratômetro de mesa Shimadzu, com correção de temperatura para $20^{\circ} \mathrm{C}$, utilizando-se de uma gota de suco puro de cada repetição, expressando-se o resultado em ${ }^{\circ}$ Brix; e) acidez total titulável (ATT): avaliada por titulometria, expressando-se o resultado em porcentagem de ácido cítrico; f) relação SS/ATT: quociente entre os dois constituintes; g) ácido ascórbico (vitamina $\mathrm{C}$ ): determinado pelo método colorimétrico com 2,4 dinitrofenilhidrazina, com os resultados expressos em $\mathrm{mg} / 100 \mathrm{~mL}$ de suco; h) podridões: frutas com características típicas de ataque de patógenos, com lesão maior ou igual a um milímetro foram consideradas deterioradas, expressando-se o resultado em porcentagem.
O delineamento experimental utilizado foi o de blocos ao acaso. A unidade experimental foi composta de quinze frutas, sendo que cada tratamento continha quatro repetições. A análise de variância dos dados foi calculada, e as médias, comparadas pelo teste de Tukey, $\mathrm{a}(\mathrm{p} \leq 0,05)$.

\section{RESULTADOS E DISCUSSÃO}

$\mathrm{Na}$ colheita das frutas, foram avaliadas as características físicas e químicas que são apresentadas na Tabela 1 .

Durante o armazenamento das frutas, as variáveis sólidos solúveis (SS), acidez total titulável (ATT) e relação SS/ATT não diferiram estatisticamente entre os tratamentos, sistemas de colheita e períodos de armazenamento. Holcroft \& Kader (1999) observaram, durante o armazenamento de morangos cv. Selva, comportamento semelhante no teor de sólidos solúveis, porém os autores observaram a diminuição da acidez durante o armazenamento. Os valores encontrados por Cordenunsi et al. (2003), ao avaliarem as mudanças físico-químicas durante o armazenamento de cinco cultivares de morango, foram semelhantes aos observados neste experimento quanto aos valores de ácido cítrico e a oscilação na relação SS/ATT.

$\mathrm{Na}$ coloração das frutas, não ocorreram diferenças estatísticas com relação à luminosidade $\left(\mathrm{L}^{*}\right)$. As frutas submetidas aos diferentes tratamentos, sistemas de colheita e períodos de armazenamento tiveram comportamento irregular quanto aos valores de $a^{*}$ e b*. A cor da superfície, representada pela pigmentação vermelho-intensa, foi indicada pelos baixos valores de ângulo Hue $\left({ }^{\circ} h\right)$, e pode ser observada nos diferentes tratamentos, sistemas de colheita e períodos de armazenamento (Tabela 2). Isto demonstra que a cor vermelha dos frutos se manteve praticamente a mesma verificada na colheita. Pérez et al. (1997) observaram que a atmosfera modificada, proporcionada por filmes plásticos, propiciou melhor coloração após o armazenamento. Segundo Calegaro et al. (2002), a manutenção da cor dos morangos durante o armazenamento é um atributo de qualidade desejado, pois o escurecimento dos frutos compromete seu aspecto visual e, portanto, sua aceitação pelo consumidor.

A firmeza da polpa das frutas oscilou entre os tratamentos. Durante o período de armazenamento, ocorreu aumento da firmeza da polpa devido à desidratação. Após três dias de armazenamento, seguido de simulação de comercialização, a firmeza da polpa das frutas, independentemente dos tratamentos, foi maior no sistema de colheita em que as mesmas receberam pré-resfriamento (Tabela 3 ). Pelayo et al. (2003) observaram, durante o armazenamento de morangos das cultivares Aromas, Diamante e Selva, que a firmeza da polpa não foi afetada durante o armazenamento na cv. Aromas, e aumentou nas demais cultivares. Porém, segundo Cordenunsi et al. (2003), a mudança na textura é uma conseqüência natural do processo de senescência e também da atmosfera em que a fruta está armazenada. Apesar das excelentes características sensoriais, o morango é muito perecível, possui limitada vida útil pós-colheita e apresenta alta taxa respiratória $\left(15 \mathrm{mg} \mathrm{CO}_{2} \mathrm{~kg}^{-1} \mathrm{~h}^{-1} \mathrm{a}^{\circ} \mathrm{C}\right.$ ), a qual aumenta entre 4 e 5 vezes quando a temperatura aumenta até $10^{\circ} \mathrm{C}$, e incrementa até 10 vezes quando a temperatura aumenta até $20^{\circ} \mathrm{C}$ (Tudela et al., 2003).

De modo geral, o teor de ácido ascórbico das frutas diminuiu durante o armazenamento e foi maior no sistema de colheita em que as mesmas receberam pré-resfriamento. Nos diferentes tratamentos, ocorreram oscilações no teor de ácido ascórbico. Wills et al. (2000) observaram a redução do teor de ácido ascórbico durante o armazenamento de morangos. Calegaro et al. (2002) também verificaram a redução do teor de ácido ascórbico durante o armazenamento de

TABELA 1 - Características físicas e químicas de morangos cv. Camarosa na colheita.

\begin{tabular}{cccccccccc}
\hline $\begin{array}{c}\text { Massa } \\
\text { Média }(\mathrm{g})\end{array}$ & SST ( ${ }^{\circ}$ Brix) & $\begin{array}{c}\text { ATT (\% ác. } \\
\text { cítrico) }\end{array}$ & $\begin{array}{c}\text { Relação } \\
\text { SST/ATT }\end{array}$ & $\mathrm{L}^{*}$ & $\mathrm{a}^{*}$ & $\mathrm{~b}^{*}$ & $\begin{array}{c}\text { ângulo } \\
\text { Hue }\end{array}$ & $\begin{array}{c}\text { Firmeza } \\
(\mathrm{N})\end{array}$ & $\begin{array}{c}\text { Ácido Ascórbico } \\
(\mathrm{mg} / 100 \mathrm{~mL})\end{array}$ \\
\hline 41,53 & 7,35 & 0,60 & 12,25 & 32,30 & 34,51 & 18,09 & 27,65 & 4,52 & 54,47 \\
\hline
\end{tabular}


TABELA 2 - Características de coloração de morangos 'Camarosa' submetidos a diferentes sistemas de colheita, tratamentos e períodos de armazenamento.

\begin{tabular}{|c|c|c|c|c|c|c|c|}
\hline \multirow{2}{*}{ Variáveis } & \multirow{2}{*}{ Trata-mentos } & \multicolumn{2}{|c|}{$\overline{\text { E1 }}$} & \multicolumn{2}{|c|}{ E2 } & \multicolumn{2}{|c|}{$\overline{E 3}$} \\
\hline & & S1 & S2 & S1 & S2 & S1 & S2 \\
\hline \multirow{4}{*}{$\mathrm{L}^{*}$} & $\mathrm{~T} 1$ & $32,91 \mathrm{aA}$ & $33,59 \mathrm{aA}$ & $30,42 \mathrm{aA}$ & $30,84 \mathrm{aA}$ & $31,53 \mathrm{aA}$ & $31,06 \mathrm{aA}$ \\
\hline & $\mathrm{T} 2$ & $32,15 \mathrm{aA}$ & $33,12 \mathrm{aA}$ & $31,30 \mathrm{aA}$ & $31,29 \mathrm{aA}$ & $31,51 \mathrm{aA}$ & $32,88 \mathrm{aA}$ \\
\hline & $\mathrm{T} 3$ & $31,87 \mathrm{aA}$ & $33,31 \mathrm{aA}$ & $31,85 \mathrm{aA}$ & $31,64 \mathrm{aA}$ & $31,57 \mathrm{aA}$ & $32,81 \mathrm{aA}$ \\
\hline & $\mathrm{T} 4$ & $33,30 \mathrm{aA}$ & $32,70 \mathrm{aA}$ & $32,41 \mathrm{aA}$ & $31,64 \mathrm{aA}$ & $33,02 \mathrm{aA}$ & $34,32 \mathrm{aA}$ \\
\hline \multirow{4}{*}{$a^{*}$} & $\mathrm{~T} 1$ & $33,54 \mathrm{aB}$ & $35,99 \mathrm{aB}$ & $35,67 \mathrm{bB}$ & $37,52 \mathrm{bA}$ & $35,31 \mathrm{aB}$ & $34,78 \mathrm{aC}$ \\
\hline & $\mathrm{T} 2$ & $33,66 \mathrm{cE}$ & $36,41 \mathrm{aB}$ & $36,54 \mathrm{bB}$ & $37,55 \mathrm{bA}$ & $34,52 \mathrm{bC}$ & $34,41 \mathrm{bD}$ \\
\hline & $\mathrm{T} 3$ & $34,05 \mathrm{bC}$ & $36,40 \mathrm{aB}$ & $36,60 \mathrm{bB}$ & $38,00 \mathrm{aA}$ & $35,79 \mathrm{aB}$ & $33,81 \mathrm{bC}$ \\
\hline & $\mathrm{T} 4$ & $35,57 \mathrm{aC}$ & $35,36 \mathrm{aC}$ & $36,73 \mathrm{aB}$ & $38,01 \mathrm{aA}$ & $36,57 \mathrm{aC}$ & $34,19 \mathrm{bD}$ \\
\hline \multirow{4}{*}{$b^{*}$} & T1 & $18,25 \mathrm{aB}$ & $19,43 \mathrm{aB}$ & $20,20 \mathrm{bB}$ & $22,62 \mathrm{aA}$ & $19,49 \mathrm{bB}$ & $18,53 \mathrm{aB}$ \\
\hline & $\mathrm{T} 2$ & $16,54 \mathrm{bC}$ & $19,34 \mathrm{aB}$ & $20,68 \mathrm{bB}$ & $22,51 \mathrm{aA}$ & $19,62 \mathrm{aA}$ & $19,04 \mathrm{aB}$ \\
\hline & $\mathrm{T} 3$ & $16,78 \mathrm{bC}$ & $19,40 \mathrm{aB}$ & $21,18 \mathrm{bB}$ & $23,17 \mathrm{aA}$ & $20,96 \mathrm{bB}$ & $16,35 \mathrm{bC}$ \\
\hline & $\mathrm{T} 4$ & $18,41 \mathrm{aB}$ & $18,08 \mathrm{aB}$ & $22,18 \mathrm{aA}$ & $22,33 \mathrm{aA}$ & $22,39 \mathrm{aA}$ & $18,12 \mathrm{aB}$ \\
\hline \multirow{4}{*}{ ângulo Hue } & $\mathrm{T} 1$ & $27,16 \mathrm{aB}$ & $28,29 \mathrm{aB}$ & $29,50 \mathrm{bB}$ & $31,05 \mathrm{bA}$ & $28,90 \mathrm{bB}$ & $27,94 \mathrm{aB}$ \\
\hline & $\mathrm{T} 2$ & $26,04 \mathrm{bC}$ & $27,97 \mathrm{aB}$ & $29,41 \mathrm{bB}$ & $30,93 \mathrm{bA}$ & $29,52 \mathrm{bB}$ & $28,89 \mathrm{aB}$ \\
\hline & $\mathrm{T} 3$ & $26,18 \mathrm{bC}$ & $28,02 \mathrm{aB}$ & $30,05 \mathrm{bB}$ & $31,36 \mathrm{aA}$ & $30,35 \mathrm{bB}$ & $25,79 \mathrm{bD}$ \\
\hline & $\mathrm{T} 4$ & $27,36 \mathrm{aC}$ & $27,04 \mathrm{aC}$ & $31,09 \mathrm{aB}$ & $30,40 \mathrm{cC}$ & $31,47 \mathrm{aA}$ & $27,90 \mathrm{aC}$ \\
\hline
\end{tabular}

Médias seguidas da mesma letra minúscula na coluna ou maiúscula na linha não diferem estatisticamente, pelo teste de Tukey $(\mathrm{p}<0,05)$. T1= controle; T2= frutas submetidas à luz UV-C; T3= frutas acondicionadas em filme de polietileno; T4= frutas submetidas à luz UV-C e acondicionadas em filme de polietileno. $\mathrm{E} 1=3$ dias a $0^{\circ} \mathrm{C}+3$ dias a $8^{\circ} \mathrm{C} ; \mathrm{E} 2=6$ dias a $0^{\circ} \mathrm{C}+3$ dias a $8^{\circ} \mathrm{C} ; \mathrm{E} 3=9$ dias a $0^{\circ} \mathrm{C}+3$ dias a $8^{\circ} \mathrm{C} . \mathrm{S} 1=$ sistema $1 ; \mathrm{S} 2=$ sistema 2.

TABELA 3 - Sólidos solúveis (SS), acidez total titulável (ATT), relação SS/ATT, firmeza da polpa e ácido ascórbico de morangos 'Camarosa' submetidos a diferentes sistemas de colheita, tratamentos e períodos de armazenamento.

\begin{tabular}{|c|c|c|c|c|c|c|c|}
\hline \multirow[b]{2}{*}{ Variáveis } & \multirow[b]{2}{*}{ Tratamentos } & \multicolumn{2}{|c|}{ E1 } & \multicolumn{2}{|c|}{ E2 } & \multicolumn{2}{|c|}{ E3 } \\
\hline & & S1 & $\mathrm{S} 2$ & S1 & $\mathrm{S} 2$ & S1 & $\mathrm{S} 2$ \\
\hline \multirow{4}{*}{ SS $\left({ }^{\circ}\right.$ Brix $)$} & $\mathrm{T} 1$ & $7,13 \mathrm{aA}$ & $6,86 \mathrm{aA}$ & $7,48 \mathrm{aA}$ & $7,42 \mathrm{aA}$ & $6,93 \mathrm{aA}$ & $6,90 \mathrm{aA}$ \\
\hline & $\mathrm{T} 2$ & $7,00 \mathrm{aA}$ & $7,27 \mathrm{aA}$ & 7,38 aA & 7,48 aA & $6,86 \mathrm{aA}$ & 7,33 aA \\
\hline & $\mathrm{T} 3$ & $6,86 \mathrm{aA}$ & $6,53 \mathrm{aA}$ & $7,48 \mathrm{aA}$ & $7,38 \mathrm{aA}$ & $6,59 \mathrm{aA}$ & $6,66 \mathrm{aA}$ \\
\hline & $\mathrm{T} 4$ & $7,33 \mathrm{aA}$ & $6,73 \mathrm{aA}$ & $7,08 \mathrm{aA}$ & $7,21 \mathrm{aA}$ & $6,76 \mathrm{aA}$ & $6,40 \mathrm{aA}$ \\
\hline \multirow{4}{*}{ ATT (\% ác. cítrico) } & $\mathrm{T} 1$ & $0,65 \mathrm{aA}$ & $0,64 \mathrm{aA}$ & $0,65 \mathrm{aA}$ & $0,67 \mathrm{aA}$ & $0,61 \mathrm{aA}$ & $0,55 \mathrm{aA}$ \\
\hline & $\mathrm{T} 2$ & $0,64 \mathrm{aA}$ & $0,67 \mathrm{aA}$ & $0,64 \mathrm{aA}$ & $0,65 \mathrm{aA}$ & $0,57 \mathrm{aA}$ & $0,64 \mathrm{aA}$ \\
\hline & $\mathrm{T} 3$ & $0,52 \mathrm{aA}$ & $0,69 \mathrm{aA}$ & $0,59 \mathrm{aA}$ & $0,60 \mathrm{aA}$ & $0,55 \mathrm{aA}$ & $0,61 \mathrm{aA}$ \\
\hline & $\mathrm{T} 4$ & $0,57 \mathrm{aA}$ & $0,64 \mathrm{aA}$ & $0,65 \mathrm{aA}$ & $0,57 \mathrm{aA}$ & $0,65 \mathrm{aA}$ & $0,60 \mathrm{aA}$ \\
\hline \multirow{4}{*}{ Relação SS/ATT } & $\overline{\mathrm{T} 1}$ & $10,82 \mathrm{aA}$ & $10,68 \mathrm{aA}$ & $11,43 \mathrm{aA}$ & $11,05 \mathrm{aA}$ & $11,42 \mathrm{aA}$ & $12,45 \mathrm{aA}$ \\
\hline & $\mathrm{T} 2$ & $10,93 \mathrm{aA}$ & $10,75 \mathrm{aA}$ & $11,68 \mathrm{aA}$ & $11,45 \mathrm{aA}$ & $11,92 \mathrm{aA}$ & $11,53 \mathrm{aA}$ \\
\hline & $\mathrm{T} 3$ & $13,17 \mathrm{aA}$ & $9,43 \mathrm{aA}$ & $12,56 \mathrm{aA}$ & $12,29 \mathrm{aA}$ & $12,11 \mathrm{aA}$ & $10,87 \mathrm{aA}$ \\
\hline & $\mathrm{T} 4$ & $12,66 \mathrm{aA}$ & $10,42 \mathrm{aA}$ & $10,98 \mathrm{aA}$ & $12,49 \mathrm{aA}$ & $10,48 \mathrm{aA}$ & $10,82 \mathrm{aA}$ \\
\hline \multirow{4}{*}{$\begin{array}{c}\text { Firmeza de Polpa } \\
(\mathrm{N})\end{array}$} & $\overline{\mathrm{T} 1}$ & $6,84 \mathrm{cE}$ & $7,15 \mathrm{bD}$ & $7,37 \mathrm{dC}$ & $9,37 \mathrm{aA}$ & $7,95 \mathrm{cB}$ & $7,41 \mathrm{cC}$ \\
\hline & $\mathrm{T} 2$ & $6,93 \mathrm{bD}$ & $8,92 \mathrm{aB}$ & $8,26 \mathrm{cC}$ & $8,57 \mathrm{cB}$ & $8,26 \mathrm{cC}$ & $9,37 \mathrm{aA}$ \\
\hline & $\mathrm{T} 3$ & $6,30 \mathrm{dD}$ & $8,52 \mathrm{aB}$ & $9,10 \mathrm{aA}$ & $9,15 \mathrm{bA}$ & 8,92 bB & $8,44 \mathrm{bC}$ \\
\hline & $\mathrm{T} 4$ & $7,10 \mathrm{aE}$ & $8,70 \mathrm{aD}$ & $9,01 \mathrm{bC}$ & $8,57 \mathrm{cD}$ & $9,59 \mathrm{aA}$ & $9,37 \mathrm{aB}$ \\
\hline \multirow{4}{*}{$\begin{array}{c}\text { Ácido ascórbico } \\
(\mathrm{mg} / 100 \mathrm{~mL} \text { de suco })\end{array}$} & $\mathrm{T} 1$ & $38,44 \mathrm{cA}$ & $40,24 \mathrm{bA}$ & $26,30 \mathrm{cB}$ & $33,05 \mathrm{abA}$ & $36,42 \mathrm{aA}$ & $35,74 \mathrm{aA}$ \\
\hline & $\mathrm{T} 2$ & $48,33 \mathrm{aB}$ & $52,83 \mathrm{aA}$ & $25,18 \mathrm{dE}$ & $25,63 \mathrm{bE}$ & $40,02 \mathrm{aC}$ & $29,00 \mathrm{bD}$ \\
\hline & $\mathrm{T} 3$ & $35,52 \mathrm{cA}$ & $27,42 \mathrm{cC}$ & $38,89 \mathrm{aA}$ & $40,24 \mathrm{aA}$ & $24,28 \mathrm{cD}$ & $29,22 \mathrm{bB}$ \\
\hline & $\mathrm{T} 4$ & $45,64 \mathrm{bA}$ & $39,79 \mathrm{bB}$ & $30,35 \mathrm{bC}$ & $40,24 \mathrm{aB}$ & $30,35 \mathrm{bC}$ & $33,05 \mathrm{aB}$ \\
\hline
\end{tabular}

Médias seguidas da mesma letra minúscula na coluna ou maiúscula na linha não diferem estatisticamente, pelo teste de Tukey $(\mathrm{p}<0,05)$. $\mathrm{T} 1=\mathrm{controle} ; \mathrm{T} 2=$ frutas submetidas à luz UV-C; $\mathrm{T} 3=$ frutas acondicionadas em filme de polietileno; $\mathrm{T} 4=$ frutas submetidas à luz UV-C e acondicionadas em filme de polietileno. $\mathrm{E} 1=3$ dias a $0^{\circ} \mathrm{C}+3$ dias a $8^{\circ} \mathrm{C} ; \mathrm{E} 2=6$ dias a $0^{\circ} \mathrm{C}+3$ dias a $8^{\circ} \mathrm{C} ; \mathrm{E} 3=9$ dias a $0^{\circ} \mathrm{C}+3$ dias a $8^{\circ} \mathrm{C}$. S1 $1=$ sistema $1 ; \mathrm{S} 2=$ sistema 2.

morangos cv. Oso Grande.

A perda de massa nas frutas acondicionadas em filmes de polietileno foi menor e não ocorreram diferenças significativas entre sistemas de colheita e períodos de armazenamento. Já nas frutas não acondicionadas em filme, observou-se aumento da perda de massa durante o armazenamento, sendo que, no sistema de colheita em que as frutas receberam pré-resfriamento, a perda de massa foi menor (Figura 2). Isto demonstra a importância da utilização de filmes de polietileno e do pré-resfriamento na redução da perda de massa de morangos durante o armazenamento. Nunes et al. (1995) observaram que frutas armazenadas em baixas temperaturas, após 6 horas da colheita, apresentaram consideráveis mudanças na coloração, na textura e no teor de água em comparação às frutas que foram imediatamente pré-resfriadas após a colheita. Segundo García et al.
(1998), a máxima perda de massa comercialmente tolerada para morangos é de $6 \%$.

A presença de podridões foi observada apenas após nove dias de armazenamento. Nas frutas submetidas ao pré-resfriamento e à luz UV-C, sendo ou não acondicionadas em filmes polietileno, não foram verificadas podridões (Figura 3). Assim, ficou demonstrada a importância da utilização do pré-resfriamento e luz UV-C durante o armazenamento de morangos. Segundo Teruel et al. (2003), o resfriamento rápido garante o consumo de um produto com melhor qualidade, aumento no tempo de comercialização e redução de perdas. Saks et al. (1996) observaram redução significativa na incidência de podridões em morangos 'Ofra', quando estes foram submetidos à luz ultravioleta. Lammertyn et al. (2003), durante o armazenamento de morangos 'Elsanta' tratados com luz UV-C, observaram a inibição no 


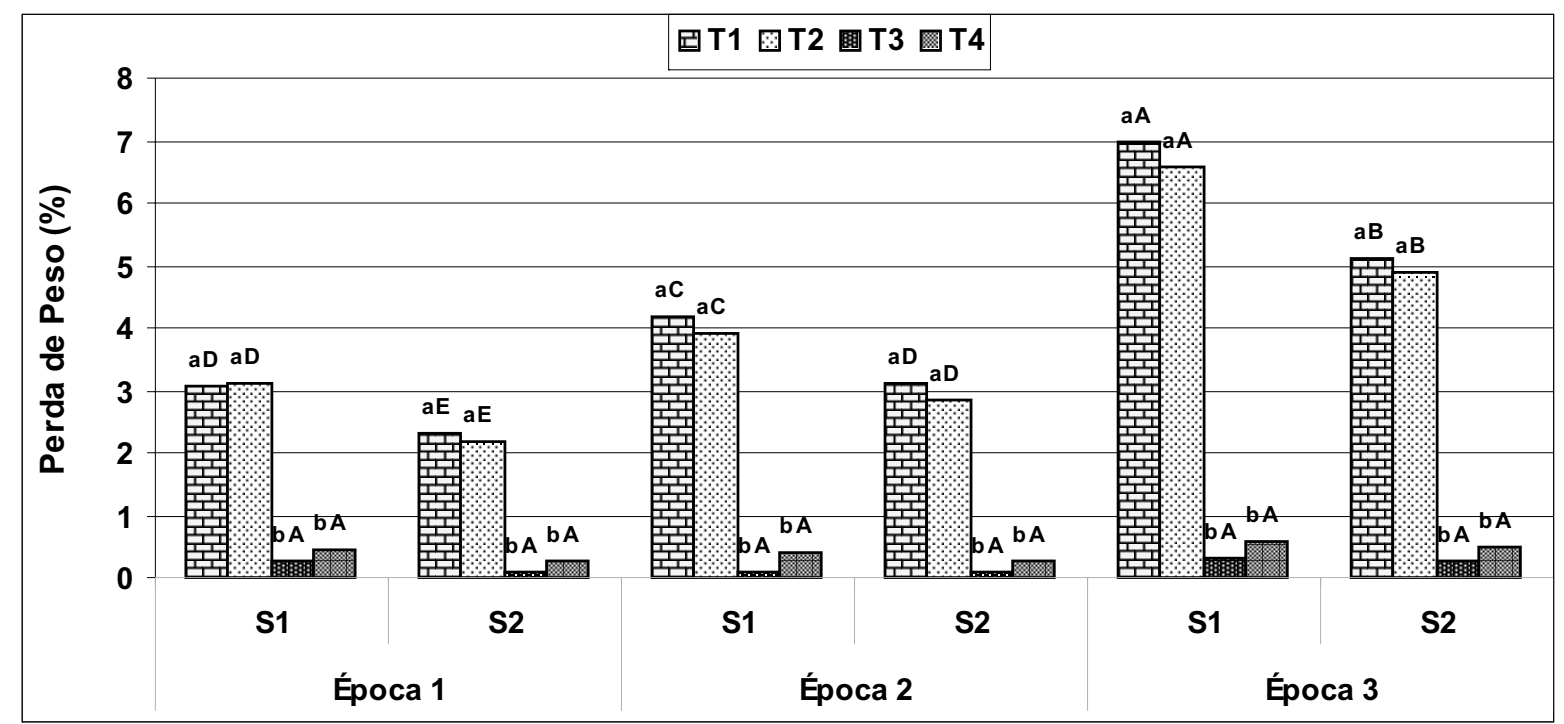

FIGURA 2 - Perda de massa (\%) de morangos cv. Camarosa submetidos a diferentes sistemas de colheita, tratamentos e períodos de armazenamento. Médias seguidas da mesma letra minúscula entre tratamentos ou maiúscula em sistemas e épocas não diferem estatisticamente, pelo teste de Tukey (p<0,05). $\mathrm{T} 1=$ controle; $\mathrm{T} 2=$ frutas submetidas à luz UV-C; $\mathrm{T} 3=$ frutas acondicionadas em filme de polietileno; $\mathrm{T} 4=$ frutas submetidas à luz UV-C e acondicionadas em filme de polietileno. $\mathrm{E} 1=3$ dias a $0^{\circ} \mathrm{C}+3$ dias a $8^{\circ} \mathrm{C} ; \mathrm{E} 2=6$ dias a $0^{\circ} \mathrm{C}+3$ dias a $8^{\circ} \mathrm{C} ; \mathrm{E} 3=9$ dias a $0^{\circ} \mathrm{C}+3$ dias a $8^{\circ} \mathrm{C}$. $\mathrm{S} 1=$ sistema $1 ; \mathrm{S} 2=$ sistema 2

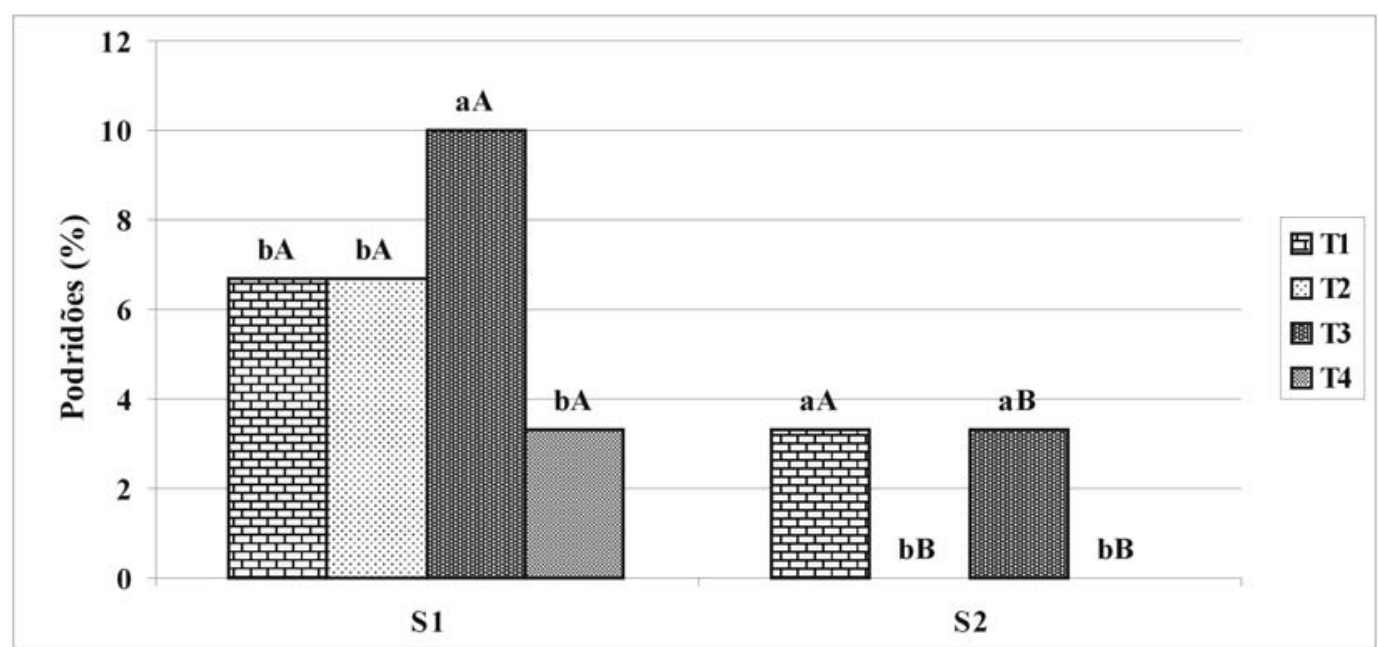

FIGURA 3 - Podridões (\%) de morangos cv. Camarosa submetidos a diferentes sistemas de colheita e tratamentos após nove dias de armazenamento a $0^{\circ} \mathrm{C}+3$ dias a $8^{\circ} \mathrm{C}$.

Médias seguidas da mesma letra minúscula entre tratamentos ou maiúscula em sistemas não diferem estatisticamente, pelo teste de Tukey (p<0,05). $\mathrm{T} 1=$ controle; T2= frutas submetidas à luz UV-C; T3= frutas acondicionadas em filme de polietileno; T4= frutas submetidas à luz UV-C e acondicionadas em filme de polietileno. $\mathrm{S} 1=$ sistema $1 ; \mathrm{S} 2=$ sistema 2 .

desenvolvimento de fungos e também a manutenção da qualidade, incluindo o aspecto visual das frutas.

\section{CONCLUSÕES}

Morangos cv. Camarosa colhidos por pessoal treinado, com utilização de luvas desinfetadas e caixas plásticas de colheita, lavadas e desinfetadas, submetidos a pré-resfriamento, com utilização de luz UV-C e filme de polietileno, mantêm a qualidade durante nove dias de armazenamento a $0^{\circ} \mathrm{C}$, mais três dias a $8^{\circ} \mathrm{C}$.

\section{REFERÊNCIAS}

BENATO, E. A. Potencial de indução de resistência em frutas póscolheita. In: ENFRUTE ENCONTRO NACIONAL SOBRE FRUTICULTURADE CLIMA TEMPERADO, 5., 2003, Fraiburgo, SC. Anais... Caçador-SC: Epagri, 2003. p. 215-220.

BENATO, E.A.; CIA, P.; SOUZA, N.L. Manejo de doenças de frutos pós-colheita. Revista Anual de Patologia de Plantas, Passo Fundo, v. 9, p. 403-440, 2001.

BINOTTI, C.S.; BENATO, E.A.; SIGRIST, J.M.M. et al. Avaliação do uso de fungicidas e UV-C combinados com atmosfera modificada em maracujá-amarelo, pós-colheita. In: CONGRESSO BRASILEIRO DEFRUTICULTURA, 17., 2002, Belém. Anais... Belém: SBF, 2002.

CALEGARO, J.M.; PEZZI, E.; BENDER, R.J. Utilização de atmosfera modificada na conservação de morangos em pós-colheita. Pesquisa Agropecuária Brasileira, Brasília, v. 37, n. 8, p. 1-6, 2002.

CORDENUNSI, B.R.; NASCIMENTO, J.R.O.; LAJOLO, F.M. Phisicochemical changes related to quality of five strawberry fruit cultivars during cool-storage. Food Chemistry, Barking, v. 83, p. 167-173. 2003.

GARCÍA, J.M.; MEDINA, R.J.; OLÍAS, J.M. Quality of strawberries automatically packed in different plastic films. Journal of Food Science, Chicago, v. 63, n. 6, p. 1037-1041, 1998.

HOLCROFT, D.M.; KADER, A.A. Carbon dioxide-induced changes in color and anthocyanin synthesis of stored strawberry fruit. HortScience, Alexandria, v. 34, n. 7, 1244-1248. 1999.

LAMMERTYN, J.; KETELAERE, B.de.; MARQUENIE, D.; MOLENBERGHS, G.; NICOLAI, B.M. Mixed models for multicategorical repeated response: modelling the time effect of physical treatments on strawberry sepal quality. Postharvest 
Biology and Technology, Waganingen, v. 30, p. 195-207, 2003.

MARQUENIE, D; MICHIELS C.W.; GEERAERD, A.H.; SCHENK, A.; SOONTJENS, C; VANIMPE, J.F.; NICOLAÝ, B.M. Using survival analysis to investigate the effect of UV-C and heat treatment on storage rot of strawberry and sweet cherry. International Journal of Food Microbiology, Amsterdan, v. 73, p. 187- 196, 2002.

NEVES, L.C.; RODRIGUES, A.C.; VIEITES, R.L. Polietileno de baixa densidade (PEBD) na conservação pós-colheita de figos cv. "Roxo de Valinhos". Revista Brasileira de Fruticultura, Jaboticabal, v. 24, n. 1, p. 57-72, 2002.

NUNES, M.C.N.; BRECHT, J.K.; MORAIS, A.M.M.B.; SARGENT, S.A. Physical and chemical-quality characterístics of strawberry after storage are reduced by a short delay to cooling. Postharvest Biology and Technology, Waganingen, v. 6, p. 17-28, 1995.

PELAYO, C.; EBELER, S.E.; KADER, A.A. Postharvest life and flavor quality of three strawberry cultivars kept at $5^{\circ} \mathrm{C}$ in air or air + Kpa $\mathrm{CO}_{2}$. Postharvest Biology and Technology, Wageningen, v. 27, p. 171-183, 2003.

PÉREZ, A.G.; SANZ, C.; OLÍAS, R.; RÍOS, J.J.; OLÍAS, J.M. Effect of modified atmosphere packaging on strawberry quality during shelf-life. In: INTERNATIONAL CONTROLLED ATMOSPHERE RESEARCHCONFERENCE, 7., 1997, Davis. Proceedings... v.3, p. 153-159.
SAKS, Y.; COPEL, A.; BARKAI-GOLAN, R. Improvement of harvested strawberry quality by illumination: colour and Botrytis infection. Postharvest Biology and Technology, Wageningen, v. 8, p. 19-27, 1996.

TERUEL, B.; CORTEZ, L.; NEVES FILHO, L. Estudo comparativo do resfriamento de laranja Valência com ar forçado e com água. Ciência e Tecnologia de Alimentos, Campinas, v. 23, n. 2, p. 174$178,2003$.

TUDELA, J.A.; VILLAESCUSA, R.; ARTÉS-HDEZ, F.; ARTÉS, F. High carbon dioxide during cold storage for keeping strawberry quality. Acta Horticulturae, Leuven, v. 600, p. 201-204, 2003.

WILLS, R.B.H.; KU, V.V.V.; LESHEM, Y.Y. Fumigation with nitric oxide to extend the postharvest life of strawberries. Postharvest Biology and Technology, Wageningen, v. 18, p. 75-79, 2000.

WSZELAKI, A.L.; MITCHAM, E.J. Effect of combinations of hot water dips, biological control and controlled atmosphere for control of gray mold on harvested strawberries. Postharvest Biology and Technology, Wageningen, v. 27, p. 255-264. 2003. 